ANNALES

POLONICI MATHEMATICI

$86.1(2005)$

\title{
Some new oscillation criteria for second order elliptic equations with damping
}

\author{
by Rong-Kun Zhuang (Huizhou) and Zheng-An YaO (Guangzhou)
}

\begin{abstract}
Some new oscillation criteria are obtained for second order elliptic differential equations with damping

$$
\sum_{i, j=1}^{n} D_{i}\left[A_{i j}(x) D_{j} y\right]+\sum_{i=1}^{n} b_{i}(x) D_{i} y+q(x) f(y)=0, \quad x \in \Omega,
$$

where $\Omega$ is an exterior domain in $\mathbb{R}^{n}$. These criteria are different from most known ones in the sense that they are based on the information only on a sequence of subdomains of $\Omega \subset \mathbb{R}^{n}$, rather than on the whole exterior domain $\Omega$. Our results are more natural in view of the Sturm Separation Theorem.
\end{abstract}

1. Introduction. In this paper, we consider the oscillation behavior of solutions of second order elliptic differential equations with damping

$$
\sum_{i, j=1}^{n} D_{i}\left[A_{i j}(x) D_{j} y\right]+\sum_{i=1}^{n} b_{i}(x) D_{i} y+q(x) f(y)=0,
$$

where $x \in \Omega$ and $\Omega$ is an exterior domain in $\mathbb{R}^{n}$. The following notations will be adopted throughout: $\mathbb{R}, \mathbb{R}^{+}$are the intervals $(-\infty, \infty),(0, \infty)$ respectively. The Euclidean length of $x$ is $|x|=\left[\sum_{i=1}^{n} x_{i}^{2}\right]^{1 / 2}$ and differentiation with respect to $x_{i}$ is denoted by $D_{i}(i=1, \ldots, n)$. For a constant $a>0$, let $S_{a}=\left\{x \in \mathbb{R}^{n}:|x|=a\right\}, G(a, \infty)=\left\{x \in \mathbb{R}^{n}:|x|>a\right\}$, $G(a, b)=\left\{x \in \mathbb{R}^{n}: a<|x|<b\right\}, G[c, b)=\left\{x \in \mathbb{R}^{n}: c \leq|x|<b\right\}$, $G(a, c]=\left\{x \in \mathbb{R}^{n}: a<|x| \leq c\right\}$. For an exterior domain $\Omega$ in $\mathbb{R}^{n}$, there exists a positive number $a_{0}$ such that $G\left(a_{0}, \infty\right) \subset \Omega$. In what follows we always assume that:

2000 Mathematics Subject Classification: 35B05, 34C10.

Key words and phrases: second order elliptic equation with damping; oscillation; generalized partial Riccati transformation; domain criteria.

Research supported by NNSF of China (No. 10171113) and NSF of Educational Department of Guangdong Province. 
$\left(\mathrm{C}_{1}\right) \quad A(x)=\left(A_{i j}(x)\right)_{n \times n}$ is a real symmetric positive definite matrix function (ellipticity condition) with $A_{i j} \in C_{\text {loc }}^{1+\mu}(\Omega), \mu \in(0,1), i, j=$ $1, \ldots, n ; \lambda_{\max }(x)$ denotes the largest (necessarily positive) eigenvalue of the matrix $A(x)$; there exists a function $\lambda \in C^{1}\left(\mathbb{R}^{+}, \mathbb{R}^{+}\right)$such that $\lambda(r) \geq \max _{|x|=r} \lambda_{\max }(x)$ for $r>0$;

$\left(\mathrm{C}_{2}\right) \quad b_{i} \in C_{\mathrm{loc}}^{1+\mu}(\Omega, \mathbb{R}), q \in C_{\mathrm{loc}}^{\mu}(\Omega, \mathbb{R}), \mu \in(0,1)$ and $q(x) \not \equiv 0$ for $|x| \geq a_{0}$; $\left(\mathrm{C}_{3}\right) \quad f \in C^{1}(\mathbb{R}, \mathbb{R}), y f(y)>0$ and $f^{\prime}(y) \geq k>0$ for all $y \neq 0$ and some constant $k$.

A function $y \in C_{\text {loc }}^{2+\mu}(\Omega, \mathbb{R}), \mu \in(0,1)$, is said to be a solution of (1.1) in $\Omega$ if $y(x)$ satisfies (1.1) for all $x \in \Omega$. For the problem of existence of solutions of (1.1), we refer the reader to the monograph [2]. We restrict our attention only to nontrivial solutions $y(x)$ of (1.1), i.e., such that $\sup \{|y(x)|: x \in \Omega\}>0$. A nontrivial solution $y(x)$ of (1.1) is called oscillatory if the zero set $\{x: y(x)=0\}$ of $y(x)$ is unbounded, otherwise it is called nonoscillatory. Equation (1.1) is called oscillatory if all its nontrivial solutions are oscillatory.

There are a great number of papers (see, for example, $[1,3,5-7,14]$ and the references quoted therein) devoted to the particular cases of (1.1), including the following second order ordinary differential equations:

$$
\begin{aligned}
y^{\prime \prime}(t)+q(t) y(t) & =0, \\
\left(r(t) y^{\prime}(t)\right)^{\prime}+q(t) y(t) & =0, \\
\left(r(t) y^{\prime}(t)\right)^{\prime}+q(t) f(y) & =0 .
\end{aligned}
$$

An important tool in the study of oscillatory behavior of solutions for (1.2) is the averaging technique. Here we list some known oscillation criteria for $(1.2)$ :

$$
\begin{gathered}
\lim _{t \rightarrow \infty} \frac{1}{t} \int_{t_{0}}^{t} \int_{t_{0}}^{s} q(v) d v d s=\infty \quad \text { (Wintner [14]); } \\
\liminf _{t \rightarrow \infty} \frac{1}{t} \int_{t_{0}}^{t} \int_{t_{0}}^{s} q(\tau) d \tau d s \\
\quad<\limsup _{t \rightarrow \infty} \frac{1}{t} \int_{t_{0}}^{t} \int_{t_{0}}^{s} q(\tau) d \tau d s \leq \infty \quad \text { (Hartman [3]) }
\end{gathered}
$$

$$
\limsup _{t \rightarrow \infty} \frac{1}{t^{m-1}} \int_{t_{0}}^{t}(t-s)^{m-1} q(s) d s=\infty \quad \text { for some } m>2
$$

(Kamenev [5]).

Some other oscillatory criteria can be found in $[8,12,16]$ and the references therein. 
For the semilinear elliptic equation

$$
\sum_{i, j=1}^{n} D_{i}\left[A_{i j} D_{j} y\right]+q(x) f(y)=0
$$

Noussair and Swanson [11] first extended the Wintner theorem by using the following partial Riccati type transformation:

$$
w(x)=-\frac{\alpha(|x|)}{f(y(x))}(A \nabla y)(x)
$$

where $\alpha \in C^{2}$ is an arbitrary positive function and $\nabla y$ denotes the gradient of $y$. Swanson [13] summarized the oscillation results for (1.8) up to 1979. For recent contributions we refer the reader to $\mathrm{Xu}$ et al. [15] and the references therein.

We see that most known oscillation criteria involve the integral of $q(x)$ and hence require the knowledge of $q(x)$ on the entire half-line $\left[a_{0}, \infty\right)$. However, from the Sturm Separation Theorem, we know that oscillation is only an interval property, i.e., if there exists a sequence of subintervals $\left[a_{i}, b_{i}\right]$ of $\left[a_{0}, \infty\right)$, with $a_{i} \rightarrow \infty$, such that for each $i$ there exists a solution of (1.2) that has at least one zero in $\left[a_{i}, b_{i}\right]$, then every solution of $(1.2)$ is oscillatory, no matter how "bad" (1.2) is (or $p$ and $q$ are) on the remaining parts of $\left[a_{0}, \infty\right)$.

Taking this into account, Kong [6] established an interval criterion for oscillation of the second order linear differential equation (1.2). Recently, $\mathrm{Li}$ and Agarwal [9, 10] and Huang [4] further studied interval oscillation criteria for nonlinear ODEs. However, for second order elliptic differential equations, whether similar results are true has remained an open question.

Motivated by the idea of Kong [6], Li and Agarwal [9, 10], Noussair and Swanson [11], and $\mathrm{Xu}$ et al. [12], in this paper we obtain, by using a generalized Riccati transformation and integral averaging technique, several new domain criteria for oscillation, that is, criteria given by the behavior of (1.1) only on a sequence of subdomains of $\Omega \subset \mathbb{R}^{n}$. Our results are extensions of the results of the above-mentioned authors.

2. Main results. We first introduce a class $\Phi$ of functions. Let $D_{0}=$ $\left\{(r, s) \in \mathbb{R}^{2}: r>s \geq a_{0}\right\}$ and $D=\left\{(r, s) \in \mathbb{R}^{2}: r \geq s \geq a_{0}\right\}$. A function $H \in C(D, \mathbb{R})$ is said to belong to $\Phi$ if there are $h_{1}, h_{2} \in C(D, \mathbb{R}), \varrho \in$ $C^{1}\left(\left[a_{0}, \infty\right), \mathbb{R}^{+}\right)$and $\eta \in C^{1}\left(\left[a_{0}, \infty\right), \mathbb{R}\right)$ satisfying the following conditions:

$$
H(r, r)=0 \text { for } r \geq a_{0} ; H(r, s)>0 \text { for all }(r, s) \in D_{0} \text {; }
$$


$\left(\mathrm{H}_{2}\right)$

$$
\begin{aligned}
& \frac{\partial}{\partial r}[H(r, s)]+\left[\frac{\varrho^{\prime}(r)}{\varrho(r)}+\frac{2 k}{\omega} \eta(r) r^{1-n}\right] H(r, s)=h_{1}(r, s) \sqrt{H(r, s)} \\
& \frac{\partial}{\partial s}[H(r, s)]+\left[\frac{\varrho^{\prime}(s)}{\varrho(s)}+\frac{2 k}{\omega} \eta(s) s^{1-n}\right] H(r, s)=-h_{2}(r, s) \sqrt{H(r, s)}
\end{aligned}
$$

For simplicity, we define functions $Q$ and $g$ as follows: For any given functions $\varrho \in C^{1}\left(\left[a_{0}, \infty\right), \mathbb{R}^{+}\right)$and $\lambda, \eta \in C^{1}\left(\left[a_{0}, \infty\right), \mathbb{R}\right)$, let

$$
\begin{aligned}
Q(r)= & \varrho(r)\left\{\int_{S_{r}}\left[q(x)-\frac{1}{4 k} B^{T} A^{-1} B-\frac{1}{2 k} \sum_{i=1}^{n} D_{i} b_{i}\right] d \sigma\right. \\
& \left.+\frac{k}{\omega} \lambda(r) \eta^{2}(r) r^{1-n}-[\lambda(r) \eta(r)]^{\prime}\right\}, \\
g(r)= & \frac{\omega}{k} \lambda(r) \varrho(r) r^{n-1},
\end{aligned}
$$

where $S_{r}=\left\{x \in \mathbb{R}^{n}:|x|=r\right\}$ for $r>0, B^{T}=\left(b_{1}(x), \ldots, b_{n}(x)\right), d \sigma$ denotes the spherical integral element in $\mathbb{R}^{n}$ and $\omega$ is the area of the unit sphere in $\mathbb{R}^{n}$.

Lemma 2.1. Let $y(x)$ be a nontrivial solution of (1.1) with $y(x)>0$ for $x \in G[c, b)$. For any $H \in \Phi$, define

$$
\begin{aligned}
W(x) & =\frac{1}{f(y)}(A \nabla y)(x)+\frac{1}{2 k} B, & & x \in G[c, b), \\
V(r) & =\varrho(r)\left[\int_{S_{r}} W(x) \gamma(x) d \sigma+\lambda(r) \eta(r)\right], & & x \in G[c, b),
\end{aligned}
$$

where $\nabla y$ denotes the gradient of $y(x)$, and $\gamma(x)=x /|x|$ for $|x| \neq 0$ is the outward unit normal to $S_{r}$. Then

$$
\frac{1}{H(b, c)} \int_{c}^{b} H(b, s) Q(s) d s \leq V(c)+\frac{1}{4 H(b, c)} \int_{c}^{b} g(s) h_{2}^{2}(b, s) d s .
$$

Proof. A direct computation with the use of (1.1) and (2.1) leads to

$$
\begin{aligned}
\operatorname{div} W(x)= & -\frac{f^{\prime}(y)}{f^{2}(y)}(\nabla y)^{T} A \nabla y-\frac{1}{f(y)}\left[q(x) f(y)+B^{T} \nabla y\right] \\
& +\frac{1}{2 k} \sum_{i=1}^{n} D_{i} b_{i} \\
\leq & -k\left[W-\frac{1}{2 k} B\right]^{T} A^{-1}\left[W-\frac{1}{2 k} B\right]-q(x) \\
& -B^{T} A^{-1}\left[W-\frac{1}{2 k} B\right]+\frac{1}{2 k} \sum_{i=1}^{n} D_{i} b_{i} \\
= & -k W^{T} A^{-1} W-q(x)+\frac{1}{4 k} B^{T} A^{-1} B+\frac{1}{2 k} \sum_{i=1}^{n} D_{i} b_{i} .
\end{aligned}
$$

Applying Green's formula to (2.2), we get 


$$
\begin{aligned}
& V^{\prime}(r)=\frac{\varrho^{\prime}(r)}{\varrho(r)} V(r)+\varrho(r)\left\{\int_{S_{r}} \operatorname{div} W(x) d \sigma+[\lambda(r) \eta(r)]^{\prime}\right\} \\
& \leq \frac{\varrho^{\prime}(r)}{\varrho(r)} V(r)-\varrho(r) k \int_{S_{r}} W^{T} A^{-1} W d \sigma \\
& \quad-\varrho(r)\left\{\int_{S_{r}}\left[q(x)-\frac{1}{4 k} B^{T} A^{-1} B-\frac{1}{2 k} \sum_{i=1}^{n} D_{i} b_{i}\right] d \sigma-[\lambda(r) \eta(r)]^{\prime}\right\} .
\end{aligned}
$$

In view of $\left(\mathrm{C}_{1}\right)$, we have $\left(W^{T} A^{-1} W\right)(x) \geq \lambda_{\max }^{-1}(x)|W(x)|^{2}$, and by the Cauchy-Schwarz inequality, we have

$$
\int_{S_{r}}|W(x)|^{2} d \sigma \geq \frac{r^{1-n}}{\omega}\left[\int_{S_{r}} W(x) \gamma(x) d \sigma\right]^{2} .
$$

Hence, by (2.2) and (2.5), we get

$$
\begin{aligned}
V^{\prime}(r) \leq & \frac{\varrho^{\prime}(r)}{\varrho(r)} V(r)-\frac{k \varrho(r) r^{1-n}}{\omega \lambda(r)}\left[\int_{S_{r}} W(x) \gamma(x) d \sigma\right]^{2} \\
& -\varrho(r)\left\{\int_{S_{r}}\left[q(x)-\frac{1}{4 k} B^{T} A^{-1} B-\frac{1}{2 k} \sum_{i=1}^{n} D_{i} b_{i}\right] d \sigma-[\lambda(r) \eta(r)]^{\prime}\right\} \\
= & \frac{\varrho^{\prime}(r)}{\varrho(r)} V(r)-\frac{k \varrho(r) r^{1-n}}{\omega \lambda(r)}\left[\frac{V(r)}{\varrho(r)}-\lambda(r) \eta(r)\right]^{2} \\
& -\varrho(r)\left\{\int_{s_{r}}\left[q(x)-\frac{1}{4 k} B^{T} A^{-1} B-\frac{1}{2 k} \sum_{i=1}^{n} D_{i} b_{i}\right] d \sigma-[\lambda(r) \eta(r)]^{\prime}\right\} \\
= & -Q(r)+\left[\frac{\varrho^{\prime}(r)}{\varrho(r)}+\frac{2 k}{\omega} \eta(r) r^{1-n}\right] V(r)-\frac{1}{g(r)} V^{2}(r) .
\end{aligned}
$$

Next we multiply $(2.6)$, with $r$ replaced by $s$, by $H(r, s)$ and integrate from $c$ to $r$, to get

$$
\begin{aligned}
\int_{c}^{r} H(r, s) & Q(s) d s \leq-\int_{c}^{r} V^{\prime}(s) H(r, s) d s-\int_{c}^{r} \frac{H(r, s)}{g(s)} V^{2}(s) d s \\
& +\int_{c}^{r}\left[\frac{\varrho^{\prime}(s)}{\varrho(s)}+\frac{2 k}{\omega} \eta(s) s^{1-n}\right] V(s) H(r, s) d s \\
= & H(r, c) V(c)-\int_{c}^{r} \frac{H(r, s)}{g(s)} V^{2}(s) d s \\
& +\int_{c}^{r}\left\{\frac{\partial H(r, s)}{\partial s}+\left[\frac{\varrho^{\prime}(s)}{\varrho(s)}+\frac{2 k}{\omega} \eta(s) s^{1-n}\right] H(r, s)\right\} V(s) d s \\
= & H(r, c) V(c)-\int_{c}^{r} \frac{H(r, s)}{g(s)} V^{2}(s) d s-\int_{c}^{r} h_{2}(r, s) \sqrt{H(r, s)} V(s) d s
\end{aligned}
$$




$$
\begin{aligned}
= & H(r, c) V(c)-\int_{c}^{r}\left[\sqrt{\frac{H(r, s)}{g(s)}} V(s)+\frac{1}{2} \sqrt{g(s)} h_{2}(r, s)\right]^{2} d s \\
& +\frac{1}{4} \int_{c}^{r} g(s) h_{2}^{2}(r, s) d s \\
\leq & H(r, c) V(c)+\frac{1}{4} \int_{c}^{r} g(s) h_{2}^{2}(r, s) d s .
\end{aligned}
$$

Let $r \rightarrow b^{-}$in (2.7). Dividing both sides by $H(b, c)$, we obtain $(2.3)$.

LEMMA 2.2. Let $y(x)$ be a nontrivial solution of (1.1) with $y(x)>0$ for $x \in G(a, c]$. For any $H \in \Phi$, let $W(r), V(r)$ be defined on $G(a, c]$ by $(2.1)$, (2.2) respectively. Then

$$
\frac{1}{H(c, a)} \int_{a}^{c} H(s, a) Q(s) d s \leq-V(c)+\frac{1}{4 H(c, a)} \int_{a}^{c} g(s) h_{1}^{2}(s, a) d s .
$$

Proof. The proof is similar to that Lemma 2.1. We multiply (2.6) by $H(s, r)$ and integrate with respect to $s$ from $r$ to $c$ for $r \in(a, c]$ to get

$$
\begin{aligned}
& \int_{r}^{c} H(s, r) Q(s) d s \\
& \leq-\int_{r}^{c} V^{\prime}(s) H(s, r) d s-\int_{r}^{c} \frac{H(s, r)}{g(s)} V^{2}(s) d s \\
& +\int_{r}^{c}\left[\frac{\varrho^{\prime}(s)}{\varrho(s)}+\frac{2 k}{\omega} \eta(s) s^{1-n}\right] V(s) H(s, r) d s \\
& =-H(c, r) V(c)-\int_{r}^{c} \frac{H(s, r)}{g(s)} V^{2}(s) d s \\
& +\int_{r}^{c}\left\{\frac{\partial H(s, r)}{\partial s}+\left[\frac{\varrho^{\prime}(s)}{\varrho(s)}+\frac{2 k}{\omega} \eta(s) s^{1-n}\right] H(s, r)\right\} V(s) d s \\
& =-H(c, r) V(c)-\int_{r}^{c} \frac{H(s, r)}{g(s)} V^{2}(s) d s+\int_{r}^{c} h_{1}(s, r) \sqrt{H(s, r)} V(s) d s \\
& =-H(c, r) V(c)-\int_{r}^{c}\left[\sqrt{\frac{H(s, r)}{g(s)}} V(s)-\frac{1}{2} \sqrt{g(s)} h_{1}(s, r)\right]^{2} d s \\
& +\frac{1}{4} \int_{r}^{c} g(s) h_{1}^{2}(s, r) d s \\
& \leq-H(c, r) V(c)+\frac{1}{4} \int_{r}^{c} g(s) h_{1}^{2}(s, r) d s .
\end{aligned}
$$

Let $r \rightarrow a^{+}$in (2.9). Dividing both sides by $H(c, a)$, we obtain $(2.8)$. 
The following theorem is an immediate consequence of Lemmas 2.1 and 2.2 .

Theorem 2.1. Assume that for some $c \in(a, b)$ and some $H \in \Phi$,

$$
\begin{aligned}
& \frac{1}{H(c, a)} \int_{a}^{c} H(s, a) Q(s) d s+\frac{1}{H(b, c)} \int_{c}^{b} H(b, s) Q(s) d s \\
& \quad>\frac{1}{4}\left(\frac{1}{H(c, a)} \int_{a}^{c} g(s) h_{1}^{2}(s, a) d s+\frac{1}{H(b, c)} \int_{c}^{b} g(s) h_{2}^{2}(b, s) d s\right) .
\end{aligned}
$$

Then every nontrivial solution of (1.1) has at least one zero in $G(a, b)=$ $\left\{x \in \mathbb{R}^{n}: a<|x|<b\right\}$

Proof. Suppose the contrary. Then without loss of generality we may assume that there is a solution $y(x)$ of (1.1) such that $y(x)>0$ for $x \in$ $G(a, b)$. From Lemmas 2.1 and 2.2, we see that both (2.3) and (2.8) hold. Adding (2.3) and (2.8), we obtain

$$
\begin{aligned}
& \frac{1}{H(c, a)} \int_{a}^{c} H(s, a) Q(s) d s+\frac{1}{H(b, c)} \int_{c}^{b} H(b, s) Q(s) d s \\
& \quad \leq \frac{1}{4}\left(\frac{1}{H(c, a)} \int_{a}^{c} g(s) h_{1}^{2}(s, a) d s+\frac{1}{H(b, c)} \int_{c}^{b} g(s) h_{2}^{2}(b, s) d s\right),
\end{aligned}
$$

which contradicts the assumption (2.10) and completes the proof.

Theorem 2.2. Suppose for each $T \geq a_{0}$ there exist $H \in \Phi$ and $a, b, c \in \mathbb{R}$ such that $T \leq a<c<b$ and (2.10) holds. Then equation (1.1) is oscillatory.

Proof. Pick a sequence $\left\{T_{i}\right\} \subset\left[a_{0}, \infty\right)$ with $T_{i} \rightarrow \infty$ as $i \rightarrow \infty$. By the assumption, for each $i \in \mathbb{N}$, there exist $a_{i}, b_{i}, c_{i} \in \mathbb{R}$ such that $T_{i} \leq a_{i}<$ $c_{i}<b_{i}$, and (2.10) holds with $a, b, c$ replaced by $a_{i}, b_{i}, c_{i}$, respectively. From Theorem 2.1, every solution $y(x)$ has at least one zero $x \in G\left(a_{i}, b_{i}\right)$. Noting that $|x|>a_{i} \geq T_{i}$ for $i \in \mathbb{N}$, we see that the zero set $\{x \in \Omega: y(x)=0\}$ of $y(x)$ is unbounded. Thus, every nontrivial solution of (1.1) is oscillatory. The proof is complete.

THEOREM 2.3. Suppose for each $l \geq a_{0}$ there exists $H \in \Phi$ such that

$$
\limsup _{r \rightarrow \infty} \int_{l}^{r}\left[H(s, l) Q(s)-\frac{1}{4} g(s) h_{1}^{2}(s, l)\right] d s>0
$$

and

$$
\limsup _{r \rightarrow \infty} \int_{l}^{r}\left[H(r, s) Q(s)-\frac{1}{4} g(s) h_{2}^{2}(r, s)\right] d s>0 .
$$

Then equation (1.1) is oscillatory. 
Proof. For any $T \geq a_{0}$, let $a=T$. In (2.12) we choose $l=a$. Then there exists $c>a$ such that

$$
\int_{a}^{c}\left[H(s, a) Q(s)-\frac{1}{4} g(s) h_{1}^{2}(s, a)\right] d s>0 .
$$

In (2.13) we choose $l=c$. Then there exists $b>c$ such that

$$
\int_{c}^{b}\left[H(b, s) Q(s)-\frac{1}{4} g(s) h_{2}^{2}(b, s)\right] d s>0 .
$$

Combining (2.14) and (2.15) we obtain (2.10). The conclusion thus comes from Theorem 2.2. The proof is complete.

From the above oscillation criteria, we can obtain various sufficient conditions for oscillation of (1.1) for different choices of $H(r, s), \varrho(s)$ and $\eta(s)$.

In Theorem 2.2, if we choose $\eta(s) \equiv 0$, and $H=H(r-s) \in \Phi$, we have $\partial H(r-s) / \partial r=-\partial H(r-s) / \partial s$; if we denote this common value by $h(r-s)$, then

$$
\begin{aligned}
& h_{1}(r, s)=\frac{h(r-s)}{\sqrt{H(r-s)}}+\frac{\varrho^{\prime}(r)}{\varrho(r)} \sqrt{H(r-s)}, \\
& h_{2}(r, s)=\frac{h(r-s)}{\sqrt{H(r-s)}}-\frac{\varrho^{\prime}(s)}{\varrho(s)} \sqrt{H(r-s)} .
\end{aligned}
$$

The subclass of $\Phi$ consisting of such $H(r-s)$ is denoted by $\Phi_{0}$. Applying Theorem 2.2 to $\Phi_{0}$, we obtain the following result.

THEOREM 2.4. Suppose for each $l \geq a_{0}$ there exists $H \in \Phi_{0}$ and $a, c \in \mathbb{R}$ such that $T \leq a<c$ and

$$
\begin{aligned}
\int_{a}^{c} H(s-a)[Q(s)+Q(2 c-s)] d s>\frac{1}{4} \int_{a}^{c}[g(s)+g(2 c-s)] \frac{h^{2}(s-a)}{H(s-a)} d s \\
+\frac{1}{2} \int_{a}^{c}\left[g(s) \frac{\varrho^{\prime}(s)}{\varrho(s)}-g(2 c-s) \frac{\varrho^{\prime}(2 c-s)}{\varrho(2 c-s)}\right] h(s-a) d s \\
+\frac{1}{4} \int_{a}^{c}\left[g(s) \frac{\varrho^{\prime 2}(s)}{\varrho^{2}(s)}+g(2 c-s) \frac{\varrho^{\prime 2}(2 c-s)}{\varrho^{2}(2 c-s)}\right] H(s-a) d s
\end{aligned}
$$

Then equation (1.1) is oscillatory.

Proof. Let $b=2 c-a$. Then $H(b-c)=H(c-a)=H((b-a) / 2)$ and for any $\phi \in L[a, b]$, we have

$$
\int_{c}^{b} \phi(s) d s=\int_{a}^{c} \phi(2 c-s) d s .
$$


Thus if (2.16) holds then (2.10) holds for $H \in \Phi_{0}, \varrho \in C^{1}\left(\left[a_{0}, \infty\right), \mathbb{R}^{+}\right)$and therefore (1.1) is oscillatory by Theorem 2.2. The proof is complete.

Define

$$
\Lambda(r)=\int_{a_{0}}^{r} \frac{s^{1-n}}{\varrho(s) \lambda(s)} d s, \quad r \geq a_{0}
$$

and let

$$
H(r, s)=[\Lambda(r)-\Lambda(s)]^{\mu} \quad(\mu>1), \quad \eta(s) \equiv 0 .
$$

Based on the above results, we obtain the following oscillation criteria of Kamenev's type.

Theorem 2.5. Assume that $\lim _{r \rightarrow \infty} \Lambda(r)=\infty$. If for each $b \geq a_{0}$, there exists $\mu>1$ such that

$$
\limsup _{r \rightarrow \infty} \frac{1}{\Lambda^{\mu-1}(r)} \int_{b}^{r}[\Lambda(s)-\Lambda(b)]^{\mu} Q(s) d s>\frac{\omega \mu^{2}}{4 k(\mu-1)}
$$

and

$$
\limsup _{r \rightarrow \infty} \frac{1}{\Lambda^{\mu-1}(r)} \int_{b}^{r}[\Lambda(r)-\Lambda(s)]^{\mu} Q(s) d s>\frac{\omega \mu^{2}}{4 k(\mu-1)},
$$

then equation (1.1) is oscillatory.

Proof. It is easy to see that

$$
\begin{aligned}
h_{1}(r, s) & =\mu[\Lambda(r)-\Lambda(s)]^{(\mu-2) / 2} \frac{r^{1-n}}{\varrho(r) \lambda(r)}, \\
h_{2}(r, s) & =\mu[\Lambda(r)-\Lambda(s)]^{(\mu-2) / 2} \frac{s^{1-n}}{\varrho(s) \lambda(s)}, \\
g(r) & =\frac{\omega}{k} \varrho(r) \lambda(r) r^{n-1} .
\end{aligned}
$$

Hence we have

$$
\begin{aligned}
\int_{b}^{r} g(s) h_{1}^{2}(s, b) d s & =\int_{b}^{r} \frac{\omega}{k} \varrho(s) \lambda(s) s^{n-1} \mu^{2}[\Lambda(s)-\Lambda(b)]^{\mu-2} \frac{\left(s^{1-n}\right)^{2}}{\varrho^{2}(s) \lambda^{2}(s)} d s \\
& =\frac{\omega \mu^{2}}{k} \int_{b}^{r}[\Lambda(s)-\Lambda(b)]^{\mu-2} \frac{s^{1-n}}{\varrho(s) \lambda(s)} d s \\
& =\frac{\omega \mu^{2}}{k(\mu-1)}[\Lambda(r)-\Lambda(b)]^{\mu-1}
\end{aligned}
$$

and

$$
\int_{b}^{r} g(s) h_{2}^{2}(r, s) d s=\int_{b}^{r} \frac{\omega}{k} \varrho(s) \lambda(s) s^{n-1} \mu^{2}[\Lambda(r)-\Lambda(s)]^{\mu-2} \frac{\left(s^{1-n}\right)^{2}}{\varrho^{2}(s) \lambda^{2}(s)} d s
$$




$$
\begin{aligned}
& =\frac{\omega \mu^{2}}{k} \int_{b}^{r}[\Lambda(r)-\Lambda(s)]^{\mu-2} \frac{s^{1-n}}{\varrho(s) \lambda(s)} d s \\
& =\frac{\omega \mu^{2}}{k(\mu-1)}[\Lambda(r)-\Lambda(b)]^{\mu-1} .
\end{aligned}
$$

Noting that $\lim _{r \rightarrow \infty} \Lambda(r)=\infty$, we have

$$
\lim _{r \rightarrow \infty} \frac{1}{4 \Lambda^{\mu-1}(r)} \int_{b}^{r} g(s) h_{1}^{2}(s, b) d s=\frac{\omega \mu^{2}}{4 k(\mu-1)}
$$

and

$$
\lim _{r \rightarrow \infty} \frac{1}{4 \Lambda^{\mu-1}(r)} \int_{b}^{r} g(s) h_{2}^{2}(r, s) d s=\frac{\omega \mu^{2}}{4 k(\mu-1)} .
$$

From (2.17) and (2.19), we have

$$
\begin{aligned}
& \lim _{r \rightarrow \infty} \frac{1}{\Lambda^{\mu-1}(r)} \int_{b}^{r}\left\{[\Lambda(s)-\Lambda(b)]^{\mu} Q(s)-\frac{1}{4} g(s) h_{1}^{2}(s, b)\right\} d s \\
& \quad=\lim _{r \rightarrow \infty} \frac{1}{\Lambda^{\mu-1}(r)} \int_{b}^{r}[\Lambda(s)-\Lambda(b)]^{\mu} Q(s) d s-\lim _{r \rightarrow \infty} \frac{1}{\Lambda^{\mu-1}(r)} \int_{b}^{r} \frac{1}{4} g(s) h_{1}^{2}(s, b) d s \\
& \quad=\lim _{r \rightarrow \infty} \frac{1}{\Lambda^{\mu-1}(r)} \int_{b}^{r}[\Lambda(s)-\Lambda(b)]^{\mu} Q(s) d s-\frac{\omega \mu^{2}}{4 k(\mu-1)}>0,
\end{aligned}
$$

i.e., (2.12) holds. Similarly, (2.18) implies (2.13) holds. From Theorem 2.3, equation (1.1) is oscillatory.

REMARK. If the assumption $\left(\mathrm{C}_{3}\right)$ is replaced by

$$
f(y) / y \geq k>0, \quad y \neq 0
$$

then we can obtain the same results when $q(x) \geq 0$ for $x \in \Omega \subset \mathbb{R}^{n}$.

ExAmple 1. Consider the second order nonlinear elliptic differential equation

$$
\begin{aligned}
\frac{\partial}{\partial x_{1}}\left[\frac{\alpha}{r^{2}} \frac{\partial y}{\partial x_{1}}\right]+ & \frac{\partial}{\partial x_{2}}\left[\frac{\beta}{r^{2}} \frac{\partial y}{\partial x_{2}}\right]+\frac{\alpha}{r^{3}} \frac{\partial y}{\partial x_{1}}+\frac{\beta}{r^{3}} \frac{\partial y}{\partial x_{2}} \\
& +\frac{(\alpha+\beta+4) r-6\left(\alpha x_{1}+\beta x_{2}\right)}{4 r^{5}}\left(y+y^{3}\right)=0
\end{aligned}
$$

where $r=\sqrt{x_{1}^{2}+x_{2}^{2}}, r \geq 1, n=2, \alpha \geq \beta>0$. It is easy to see that

$$
\lambda(r)=\frac{\alpha}{r^{2}}, \quad q(x)=\frac{(\alpha+\beta+4) r-6\left(\alpha x_{1}+\beta x_{2}\right)}{4 r^{5}}, \quad f(y)=y+y^{3} .
$$


Let $\varrho=1$. Then

$\Lambda(r)=\int_{1}^{r} \frac{s^{1-n}}{\lambda(s)} d s=\frac{1}{2 \alpha}\left(r^{2}-1\right), \quad Q(r)=\frac{2 \pi}{r^{3}}, \quad f^{\prime}(y)=1+3 y^{2} \geq 1, \quad \omega=2 \pi$.

Then, for any $b \geq 1$,

$$
\begin{gathered}
\lim _{r \rightarrow \infty} \frac{1}{\Lambda^{\mu-1}(r)} \int_{b}^{r}[\Lambda(s)-\Lambda(b)]^{\mu} Q(s) d s=\lim _{r \rightarrow \infty} \frac{[\Lambda(r)-\Lambda(b)]^{\mu} \cdot 2 \pi / r^{3}}{(\mu-1) \Lambda^{\mu-2} r / \alpha} \\
=\frac{2 \pi \alpha}{\mu-1} \lim _{r \rightarrow \infty} \frac{[\Lambda(r)-\Lambda(b)]^{\mu}}{\Lambda^{\mu-2} r^{4}}=\frac{2 \pi}{4(\mu-1) \alpha} .
\end{gathered}
$$

For any $\alpha<1$, there exists $\mu>1$ such that $\frac{2 \pi}{4(\mu-1) \alpha}>\frac{2 \pi \mu^{2}}{4(\mu-1)}$, so $(2.17)$ holds.

Noting that

$$
\begin{aligned}
\int_{b}^{r}\left\{[\Lambda(r)-\Lambda(s)]^{\mu}-\right. & {\left.[\Lambda(s)-\Lambda(b)]^{\mu}\right\} \frac{2 \pi}{s^{3}} d s } \\
\geq & \frac{2 \pi}{r^{3}} \int_{b}^{r}\left\{[\Lambda(r)-\Lambda(s)]^{\mu}-[\Lambda(s)-\Lambda(b)]^{\mu}\right\} d s=0,
\end{aligned}
$$

we get

$$
\int_{b}^{r}[\Lambda(r)-\Lambda(s)]^{\mu} Q(s) d s \geq \int_{b}^{r}[\Lambda(s)-\Lambda(b)]^{\mu} Q(s) d s
$$

Hence

$$
\begin{aligned}
\lim _{r \rightarrow \infty} \frac{1}{\Lambda^{\mu-1}(r)} \int_{b}^{r}[\Lambda(r)-\Lambda(s)]^{\mu} & Q(s) d s \\
& \geq \lim _{r \rightarrow \infty} \frac{1}{\Lambda^{\mu-1}(r)} \int_{b}^{r}[\Lambda(s)-\Lambda(b)]^{\mu} Q(s) d s \\
& =\frac{2 \pi}{4(\mu-1) \alpha} .
\end{aligned}
$$

This means that (2.18) holds for the same $\mu>1$. Applying Theorem 2.5, we find that equation (2.21) is oscillatory for $\alpha<1$.

Acknowledgments. The authors are thankful to the referee for his valuable comments and suggestions.

\section{References}

[1] W. B. Fite, Concerning the zeros of the solutions of certain differential equations, Trans. Amer. Math. Soc. 19 (1918), 341-352. 
[2] D. Gilbarg and N. S. Trudinger, Elliptic Partial Differential Equations of Second Order, Springer, New York, 1983.

[3] P. Hartman, On nonoscillatory linear differential equations of second order, Amer. J. Math. 74 (1952), 389-400.

[4] C. C. Huang, Oscillation and nonoscillation for second order linear differential equations, J. Math. Anal. Appl. 210 (1997), 712-723.

[5] I. V. Kamenev, An integral criterion for oscillation of linear differential equations of second order, Mat. Zametki 23 (1978), 249-251 (in Russian).

[6] Q. Kong, Interval criterion for oscillation of second order linear differential equations, J. Math. Anal. Appl. 229 (1999), 258-270.

[7] W. Leighton, The detection of the oscillation of solutions of a second order linear differential equation, Duke Math. J. 17 (1950), 57-61.

[8] H. J. Li, Oscillation criteria for second order linear differential equations, J. Math. Anal. Appl. 194 (1995), 217-234.

[9] W. T. Li and R. P. Agarwal, Interval oscillation criteria related to integral averaging technique for certain nonlinear differential equations, ibid. 245 (2000), 171-188.

[10] - - - Interval oscillation criteria for second-order nonlinear differential equations with damping, Comput. Math. Appl. 40 (2000), 217-230.

[11] E. S. Noussair and C. A. Swanson, Oscillation of semilinear elliptic inequalities by Riccati transformations, Canad. J. Math. 32 (1980), 908-923.

[12] Ch. G. Philos, Oscillation theorems for linear differential equations of second order, Arch. Math. (Basel) 53 (1989), 483-492.

[13] C. A. Swanson, Semilinear second-order elliptic oscillation, Canad. Math. Bull. 22 (1979), 139-157.

[14] A. Wintner, A criterion of oscillatory stability, Quart. J. Appl. Math. 7 (1949), $115-117$.

[15] Z. T. Xu, B. G. Jia and D. K. Ma, Oscillation theorem for elliptic equations with damping, Appl. Math. Comput. 156 (2004), 93-106.

[16] J. Yan, Oscillation theorems for second order linear differential equations with damping, Proc. Amer. Math. Soc. 98 (1986), 276-282.

Department of Mathematics

Huizhou University

Huizhou, Guangdong 516015, P.R. China

E-mail: gdzrk@sina.com, zhuangrk@hzu.edu.cn
Department of Mathematics Sun Yat-Sen (Zhongshan) University Guangzhou 510275, P.R. China 